\title{
Generalized extended Weibull power series family of distributions
}

\author{
Said H. Alkarni \\ Department of Quantitative Analysis
}

\begin{abstract}
In this study, we introduce a new family of models for lifetime data called generalized extended Weibull power series family of distributions by compounding generalized extended Weibull distributions and power series distributions. The compounding procedure follows the same setup carried out by Adamidis (1998). The proposed family contains all types of combinations between truncated discrete with generalized and non-generalized Weibull distributions. Some existing power series and subclasses of mixed lifetime distributions become special cases of the proposed family, such as the compound class of extended Weibull power series distributions proposed by Silva et al. (2013) and the generalized exponential power series distributions introduced by Mahmoudi and Jafari (2012). Some mathematical properties of the new class are studied, including the cumulative distribution function, density function, survival function, and hazard rate function. The method of maximum likelihood is used for obtaining a general setup for estimating the parameters of any distribution in this class. An expectation-maximization algorithm is introduced for estimating maximum likelihood estimates. Special subclasses and applications for some models in a real dataset are introduced to demonstrate the flexibility and the benefit of this new family.
\end{abstract}

Key words: generalized extended Weibull power series distributions, Weibull power series distribution, generalized power series distributions

\section{Introduction}

The modeling and analysis of lifetimes is an important aspect of statistical work in a wide variety of scientific and technological fields, such as public health, actuarial science, biomedical studies, demography, and industrial reliability. In risk modeling, the lifetime associated with a particular risk is not observable, as only the maximum or the minimum lifetime value among all the risks can be observed. In reliability, we observe only the maximum component lifetime of a parallel system and the cause of failure. Lifetime data modeling is introduced by compounding any continuous distribution and power series distributions. The Weibull distribution is exhaustively used for describing hazard rates due to its negatively and positively skewed density shapes. Chahkandi and Ganjali (2009) proposed the exponential power series family of distributions that generalize into a two-parameter exponential power series called the Weibull 
power series (WPS) class of distributions by Morais and Barreto-Souza (2011). The WPS distributions can have an increasing, decreasing, and upside-down bathtub failure rate function. In the same manner, the exponentiated Weibull power series distribution and its applications were presented by Mahmoudi and Shiran (2012). Recently, the exponentiated Weibull-Poisson distribution and its applications were introduced by Mahmoudi and Sepahdar (2013). The generalized exponential power series (GEPS) distributions were proposed by Mahmoudi and Jafari (2012), following the same approach developed by Morais and Barreto-Souza (2011) by compounding the generalized exponential and the power series distributions. Leahu et al. (2013) proposed the power series distributions in the lifetime as the maximum or minimum of the sample with a power series distributed size. The complementary exponential power series distribution with increasing failure rate was introduced by Flores et al. (2013) as a complement to the exponential power series model proposed by Chahkandy and Ganjali (2009). Silva et al. (2013) introduced the power series distributions of the compound class of the extended Weibull distribution. Recently, Bourguignon et al. (2014) proposed a new class of fatigue life distribution as the Birnbaum -Saunders power series class of distributions. We combine the GEPS distributions introduced by Mahmoudi and Jafari (2012) and the compound class of the extended Weibull power series distributions (EWPS) proposed by Silva et al. (2013) into a more general family called the generalized extended Weibull power series (GEWPS).

This study aims to generalize the EWPS distributions to obtain a new and more flexible family to describe reliability data. The proposed family can be applied to other fields, including business, environment, actuarial science, biomedical studies, demography and industrial reliability, and many other fields. This family contains several subclasses and lifetime models as special cases. In addition, it gives us flexibility of choosing any compound lifetime distribution (generalized and not generalized) for modeling any type of lifetime data.

Consider a system with $N$ components, where $N$ (the number of components) is a discrete random variable with support $\{1,2, \ldots\}$. The lifetime of the $i$ th $(1,2, \ldots, N)$ component is the nonnegative continuous random variable, say $X_{i}$, the distribution of which belongs to one of the lifetime distributions, such as exponential, gamma, Weibull, and Pareto, among others. The discrete random variable $N$ can have several distributions, such as zero-truncated Poisson, geometric, binomial, logarithmic, and the power series distributions in general. The non-negative random variable $X$ denoting the lifetime of such a system is defined by $X=\min \left\{X_{i}\right\}$ or $X=\min \left\{X_{i}\right\}$ based on whether the components are series or parallel. By taking a system with parallel components in which the random variable $N$ has the power series distributions and the random variable $X_{i}$ follows the generalized Weibull distribution, we introduce the GEWPS class of distributions that contain the GEPS and the EWPS distributions as special cases.

This paper is organized as follows. In Section 2, we define the class of Weibull and generalized Weibull distributions, and demonstrate the many existing models that can be deduced as special cases of the proposed unified model. In Section 3, we define the GEWPS class of distributions in terms of distribution functions and special cases of some existing classes. In Section 4, we provide the general properties of the GEWPS class, including the densities and the 
survival and hazard rate functions. Quantiles, moment generating function, and order statistics of GEWPS are discussed in Section 5. The estimation of the GEWPS parameters is investigated in Section 6 using the maximum likelihood method with expectation-maximization (EM) algorithm and a large sample inference. In Section 7, special subclasses and some special distributions are introduced along with the flexible mathematical forms of their properties. In Section 8, two models are presented and applied to illustrate how to use the proposed family. Finally, some concluding remarks are addressed in Section 9.

\section{The class of Weibull and Generalized Weibull distribution}

company promises to pay claims to the insureds if some defined events (injury, accident, death, etc.) occur. However in many cases, claims originating in a particular year are often settled with a time delay of years or perhaps decades. Therefore, a method to estimate the expected liability is needed so that the insurer can calculate the profit of written policies, and allocate reserved assets to ensure liquidity. Since loss reserves generally represent by far the largest liability, and the greatest source of financial uncertainty in an insurance company, an appropriate valuation of insurance liabilities including risk margin is one of the most important issues for a general insurer. Risk margin is the component of the value of claims liability that relates to the inherent uncertainty.

Weibull distribution is one of the most widely used lifetime distributions in terms of reliability. A large number of modifications have been suggested for the Weibull distribution to improve the shape of the hazard rate function. Peng and Yan (2014) presented many references on this matter. The class of extended Weibull distributions (EW) was proposed by Gurvich et al. (1997). This class is illustrated by the following definition.

Definition 1: A random variable $X_{i}$ is a member of the Weibull class of distribution if its cumulative distribution function (cdf) is given by

$$
G_{W}(x ; \alpha, \xi)=G_{W}(x)=1-e^{-\alpha H(x ; \xi)} ; x, \alpha>0,
$$

where $\mathrm{H}(x ; \xi)=\mathrm{H}(\mathrm{x})$ is a non-negative monotonically increasing function that depends on the

parameter vector $\xi>0$. The corresponding probability density function (pdf) becomes

$$
g_{W}(x ; \alpha, \xi)=g_{W}(x)=\alpha h(x) e^{-\alpha H(x)} ; x, \alpha>0,
$$

where $\mathrm{h}(\mathrm{x})=\mathrm{h}(x ; \xi)$ is the first derivative of $\mathrm{H}(x)$. The distributions of most Weibull types can be re-written in form (1) depending on the choice of the function $\mathrm{H}(x)$. Silva et al. (2013) 
and Gurvich et al. (1997) listed some examples for this class. By using the idea of Gupta and Cundu (1999), the generalized exponential of this class can be modified as follows:

Definition 2: A random variable $X_{i}$ belongs to the generalized extended Weibull distribution class if its cdf is given by

$$
G(x ; \alpha, \delta, \xi)=G(x)=\left(1-e^{-\alpha H(\mathrm{x})}\right)^{\delta} ; x, \alpha, \delta>0,
$$

where $\mathrm{H}(x)$ is a non-negative monotonically increasing function that depends on a parameter vector $\xi>0$. The corresponding pdf becomes

$$
g(x ; \alpha, \delta, \xi)=g(x)=\alpha \delta h(x) e^{-\alpha H(x)}\left(1-e^{-\alpha H(x)}\right)^{\delta-1} ; x, \alpha, \delta>0,
$$

where $\mathrm{h}(x)$ is the first derivative of $\mathrm{H}(x)$. One can see that $G(x)=\left(G_{W}(x)\right)^{\delta}$, and thus $g(x)=\delta\left(G_{W}(x)\right)^{\delta-1} g_{W}(x)$. The distributions of most Weibull and exponentiated Weibull types can be written in form (3) depending on the choice of the function $\mathrm{H}(x)$ and $\delta$. Table 1 displays useful $H(x)$ and corresponding parameter vectors for some existing distributions.

Table 1:Special distributions and the corresponding $\mathrm{H}(x ; \xi)$ and vector $\xi$.

\begin{tabular}{lccccl}
\hline \multicolumn{1}{c}{ Distribution } & $H(x)$ & $\alpha$ & $\delta$ & $\xi$ & Reference \\
\hline Exponential & $x$ & $\alpha$ & 1 & - & Johnson et al. (1994) \\
Exponential power & $e^{(\lambda x)^{\beta}-1}$ & $\alpha$ & 1 & {$[\lambda, \beta]$} & Smith and Bain (1975) \\
Burr XII $(x \geq 0)$ & $\log \left(1+x^{c}\right)$ & $\alpha$ & 1 & $\mathrm{c}$ & Rodriguez (1977) \\
Weibull $(x \geq 0)$ & $x^{\gamma}$ & $\alpha$ & 1 & $\gamma$ & Johnson et al. (1994) \\
Modified Weibull & $x^{\gamma} e^{\lambda x}$ & $\alpha$ & 1 & {$[\gamma, \lambda]$} & Lai et al. (2003) \\
Weibull extension & $\lambda\left[e^{(x / \lambda)^{\beta}}-1\right]$ & $\alpha$ & 1 & {$[\lambda, \beta]$} & Xie et al. (2002) \\
Exponential power & $\exp \left[(\lambda x)^{\beta}\right]-1$ & $\alpha$ & 1 & {$[\beta, \lambda]$} & Smith and Bain (1975) \\
Pareto $(x \geq k)$ & $\log (x / k)$ & $\alpha$ & 1 & $\mathrm{k}$ & Johnson et al. (1994) \\
Generalized exponential & $x$ & $\alpha$ & $\delta$ & - & Gupta and Kundu (2000) \\
Exponentiated Weibull & $\alpha^{\gamma-1} x^{\gamma}$ & $\alpha$ & $\delta$ & $\gamma$ & Nassar and Eissa (2003) \\
Exp. Mod. Weibull ext. & $\lambda\left[1-e^{(x / \lambda)^{\beta}}\right]$ & $\alpha$ & $\delta$ & {$[\lambda, \beta]$} & Sarhan and Apaloo (2013) \\
Exponentiated Rayleigh & $x^{2}$ & $\alpha$ & $\delta$ & - & Surles and Padgett (2001) \\
\hline
\end{tabular}

\section{The GEWPS family}


In this section, we derive the family of GEWPS distributions by compounding the generalized extended Weibull class and power series distributions.

Let $N$, be a zero truncated discrete random variable having a power series distribution with the following probability mass function:

$$
p_{n}=p(N=n)=\frac{a_{n} \theta^{n}}{c(\theta)}, n=1,2, \ldots,
$$

where $a_{n} \geq 0$ depends only on $n, c(\theta)=\sum_{n=1}^{\infty} a_{n} \theta^{n}$, and $\theta \in(0, s)$ is chosen in such a way that $c(\theta)$ is finite. Given $N$, let $X_{1}, \cdots, X_{N}$ be independent and identically distributed (iid) random variables following (3). Let $X_{(n)}=\max \left\{X_{i}\right\}_{i=1}^{N}$. Then, the cdf of $X_{(n)} \mid N=n$ is given by

$$
G_{\mathrm{X}(n) \mid N=n}(\mathrm{X})=\left[1-e^{-\alpha H(x)}\right]^{n \delta}, \mathrm{X}>0, \mathrm{n} \geq 1 .
$$

That is, $X_{(n)} \mid N=n$ has a generalized extended Weibull class of distribution with parameters $\theta, \delta, \alpha$ and $\xi$ based on the same function $\mathrm{H}(x)$. The generalized power series distribution denoted by GEWPS with an increasing failure rate is defined by the marginal distribution (cdf) of $X_{(n)}$ :

$$
F_{X_{(n)}}(x)=\sum_{n=1}^{\infty} \frac{a_{n} \theta^{n}}{c(\theta)}\left(1-e^{-\alpha H(x)}\right)^{\delta n}=\frac{c\left(\theta\left(1-e^{-\alpha H(x)}\right)^{\delta}\right)}{c(\theta)}, \quad \mathrm{x}>0,
$$

which can be written as

$$
F_{X_{(n)}}(x)=\sum_{n=1}^{\infty} p_{n}(G(x))^{n}=\frac{c(\theta G(x))}{c(\theta)}, \mathrm{x}>0 .
$$

Note that if $\mathrm{H}(x)=\mathrm{x}$, the model is reduced to the GEPS introduced by Mahmoudi and Jafari (2012).

Remarks. Let $X_{(1)}=\min \left\{X_{i}\right\}_{i=1}^{N}$, then

(1) The cdf of $X_{(1)}$ is

$$
F_{X_{(1)}}(x)=1-\frac{c(\theta-\theta G(x))}{c(\theta)}=1-\frac{c(\theta(1-G(x)))}{c(\theta)} .
$$

Note that if $\delta=1$, then the cdf of $X_{(1)}$ is:

$$
F_{X_{(1)}}(x)=1-\frac{c\left(\theta\left(1-G_{W}(x)\right)\right)}{c(\theta)}=1-\frac{c\left(\theta e^{-\alpha H(x)}\right)}{c(\theta)},
$$

which is called the EWPS proposed by Silva et al. (2013). 
If $\delta=1$ and if $H(x)=x$, then the cdf of $X_{(1)}$ becomes

$$
F_{X_{(1)}}(x)=1-\frac{c\left(e^{-\alpha x}\right)}{c(\theta)},
$$

which is the exponential power series distributions developed by Chahkandi and Ganjali (2009) that include the lifetime distributions class proposed by Adamidis and Lukas (1998), Kus (2007), Tahmasbi and Rezaei (2008).

(2) Let $Y=G^{-1}(1-G(X))$, where $G^{-1}$ is the inverse function of $G$, then $Y$ has a GEWPS distribution as

$$
\begin{aligned}
F_{Y}(y) & =P(Y \leq y)=P\left(G^{-1}(1-G(X)) \leq y\right)=P\left(X \geq G^{-1}(1-G(y))\right) \\
& =1-F_{X_{(1)}}\left(G^{-1}(1-G(y))\right)=\frac{c(\theta G(y))}{c(\theta)} .
\end{aligned}
$$

Based on the choice of $a_{n}, c(\theta), H(\mathrm{x})$ and $\delta$ with form (6), this class covers the entire compound truncated discrete distributions with all of the continuous lifetime distributions in the literature.

\section{Density, survival, and hazard functions}

The probability density functions associated with (7) and (8), respectively, are given by

$$
\begin{aligned}
f_{X_{(n)}}(x) & =\theta \mathrm{g}(\mathrm{x}) \frac{c^{\prime}(\theta \mathrm{G}(\mathrm{x}))}{c(\theta)} \\
& =\theta \alpha \delta h(x) e^{-\alpha H(x)}\left(1-e^{-\alpha H(x)}\right)^{\delta-1} \frac{c^{\prime}\left(\theta\left(1-e^{-\alpha H(x)}\right)^{\delta}\right)}{c(\theta)}
\end{aligned}
$$

and

$$
\begin{aligned}
f_{X_{(1)}}(x) & =\theta \mathrm{g}(\mathrm{x}) \frac{c^{\prime}(\theta(1-G(x)))}{c(\theta)} \\
& =\theta \alpha \delta h(x) e^{-\alpha H(x)}\left(1-e^{-\alpha H(x)}\right)^{\delta-1} \frac{c^{\prime}\left(\theta\left(1-\left(1-e^{-\alpha H(x)}\right)^{\delta}\right)\right)}{c(\theta)} .
\end{aligned}
$$

The survival functions are given by

$$
s_{X_{(n)}}(x)=1-\frac{c(\theta G(x))}{c(\theta)}=1-\frac{c\left(\theta\left(1-e^{-\alpha H(x)}\right)^{\delta}\right)}{c(\theta)}
$$

And

$$
s_{X_{(1)}}(x)=\frac{c(\theta(1-G(x))}{c(\theta)}=\frac{c\left(\theta\left(1-\left(1-e^{-\alpha H(x)}\right)^{\delta}\right)\right)}{c(\theta)} .
$$

The corresponding hazard rate functions are 


$$
\begin{aligned}
\tau_{X_{(n)}}(x) & =\frac{f_{X_{(n)}}(\mathrm{x})}{s_{X_{(n)}}(x)}=\theta g(x) \frac{c^{\prime}(\theta G(x))}{c(\theta)-c(\theta G(x))} \\
& =\theta \alpha \delta h(x) e^{-\alpha H(x)}\left(1-e^{-\alpha H(x)}\right)^{\delta-1} \frac{c^{\prime}\left(\theta\left(1-e^{-\alpha H(x)}\right)^{\delta}\right)}{c(\theta)-c\left(\theta\left(1-e^{-\alpha H(x)}\right)^{\delta}\right)}
\end{aligned}
$$

and

$$
\begin{aligned}
\tau_{X_{(1)}}(x) & =\frac{f_{X_{(1)}}(\mathrm{x})}{s_{X_{(1)}}(x)}=\theta \mathrm{g}(\mathrm{x}) \frac{c^{\prime}(\theta(1-G(x)))}{c(\theta(1-G(x)))} \\
& =\theta \alpha \delta h(x) e^{-\alpha H(x)}\left(1-e^{-\alpha H(x)}\right)^{\delta-1} \frac{c^{\prime}\left(\theta\left(1-\left(1-e^{-\alpha H(x)}\right)^{\delta}\right)\right)}{c\left(\theta\left(1-\left(1-e^{-\alpha H(x)}\right)^{\delta}\right)\right)} .
\end{aligned}
$$

The limiting distribution of the GPS when $\theta \rightarrow 0^{+}$is

$$
\begin{gathered}
\lim _{\theta \rightarrow 0^{+}} F_{X_{(n)}}(x)=\lim _{\theta \rightarrow 0^{+}} \frac{c(\theta G(x))}{c(\theta)}=\lim _{\theta \rightarrow 0^{+}} \frac{\sum_{n=1}^{\infty} a_{n} \theta^{n}(G(x))^{n}}{\sum_{n=1}^{\infty} a_{n} \theta^{n}} \\
=\lim _{\theta \rightarrow 0^{+}} \frac{a_{c}(G(x))^{c}+\sum_{n=c+1}^{\infty} a_{n} \theta^{n-c}(G(x))^{n}}{a_{c}+\sum_{n=c+1}^{\infty} a_{n} \theta^{n-c}} \\
=(G(x))^{c}=\left(1-e^{-\alpha H(x)}\right)^{c \delta}
\end{gathered}
$$

where $c=\min \left\{\mathrm{n} \in \mathrm{N}, a_{n}>0\right\}$.

The densities of GEWPS can be expressed as an infinite number of linear combinations of densities of the order statistics. Given that $c^{\prime}(\theta)=\sum_{n=1}^{\infty} n a_{n} \theta^{n-1}$, therefore,

$$
f_{X_{(n)}}(x)=\theta \mathrm{g}(\mathrm{x}) \frac{c^{\prime}(\theta G(\mathrm{x}))}{c(\theta)}=\sum_{n=1}^{\infty} p(\mathrm{~N}=\mathrm{n}) \mathrm{g}_{Y_{(n)}}(x ; n),
$$

where $\mathrm{g}_{Y_{(n)}}(x ; n)$ is the density function of $Y_{(n)}=\max \left(Y_{1}, \ldots, Y_{n}\right)$, given by

$$
\mathrm{g}_{Y_{(n)}}(x ; n)=n g(x)(G(x))^{n-1}=n \alpha \delta h(x ; \eta) e^{-\alpha H(x)}\left(1-e^{-\alpha H(x)}\right)^{n \delta-1}
$$

Moreover,

$$
f_{X_{(1)}}(x)=\theta \mathrm{g}(\mathrm{x}) \frac{c^{\prime}(\theta-\theta G(\mathrm{x}))}{c(\theta)}=\sum_{n=1}^{\infty} p(\mathrm{~N}=\mathrm{n}) \mathrm{g}_{Y_{(1)}}(x ; n),
$$


where $\mathrm{g}_{Y_{(1)}}(x ; n)$ is the density function of $Y_{(1)}=\min \left(Y_{1}, \ldots, Y_{n}\right)$, given by

$$
g_{X_{(1)}}(x ; n)=n g(x)(1-G(x))^{n-1}=n \alpha \delta h(x) e^{-\alpha \delta(n-1) H(x)}\left(1-e^{-\alpha H(x)}\right)^{\delta-1}
$$

\section{Quantiles, moments, and order statistics}

Let $X$ be a random variable with cdf as in (7). The quantile function, i.e., $Q_{X_{n}}(p)$, is defined by $F_{X_{(n)}}\left(Q_{X_{(n)}}(\mathrm{p})\right)=\mathrm{p}, \mathrm{p} \in(0,1)$. Therefore,

$$
Q_{X_{(\mathrm{n})}}(p)=H^{-1}\left\{-\frac{1}{\alpha} \log \left[\left(1-\frac{c^{-1}((\mathrm{p} c(\theta))}{\theta}\right)^{\frac{1}{\delta}}\right]\right\},
$$

with the cdf as in (8)

$$
Q_{X_{(1)}}(p)=H^{-1}\left\{-\frac{1}{\alpha} \log \left[1-\left(1-\frac{c^{-1}((1-p) c(\theta))}{\theta}\right)^{\frac{1}{\delta}}\right]\right\},
$$

where $\theta, \alpha>0, p \in(0,1)$.

The moment generating function is obtained as follows:

$$
\begin{aligned}
& M_{X_{(n)}}(t)=\int_{0}^{\infty} e^{t x} f_{X(n)}(x) d x=\int_{0}^{\infty} e^{t x} \sum_{n=1}^{\infty} P(N=n) g_{X(n)}(x) d x \\
& =\sum_{n=1}^{\infty} P(N=n) \int_{0}^{\infty} e^{t x} g_{X(n)}(x) d x=\sum_{n=1}^{\infty} P(N=n) E\left(\mathrm{Y}_{(n)}^{k}\right),
\end{aligned}
$$

which can be obtained in the function $\mathrm{H}(x)$. Similarly,

$$
M_{X_{(1)}}(t)=\int_{0}^{\infty} e^{t x} f_{X(1)}(x) d x=\sum_{n=1}^{\infty} P(N=n) \int_{0}^{\infty} e^{t x} g_{X(1)}(x) d x=\sum_{n=1}^{\infty} P(N=n) E\left(\mathrm{Y}_{(1)}^{k}\right) .
$$

Order statistics is among the most fundamental tools in non-parametric statistics and inference. It enters estimation problems and hypothesis tests in many ways. The probability distribution function of the ith order statistics from a random sample $X_{1}, \ldots, X_{m}$ with density function (8) is given by

$$
f_{i: m}^{X_{(n)}}(x)=\frac{m !}{(i-1) !(m-i) !} f_{X_{(n)}}(x)\left[\frac{c(\theta G(x))}{c(\theta)}\right]^{i-1}\left[1-\frac{c(\theta G(x))}{c(\theta)}\right]^{m-i}, x>0 .
$$


Using the binomial expansion, the above formula can be written as follows:

$$
f_{i: m}^{X_{(n)}}(x)=\frac{m !}{(i-1) !(m-i) !} f_{X_{(n)}}(x) \sum_{j=0}^{m-i}(-1)^{j}\left(\begin{array}{c}
m-i \\
j
\end{array}\right)\left[\frac{c(\theta G(x))}{c(\theta)}\right]^{m-j-1}, x>0 .
$$

For the density function in (9),

$$
f_{i: m}^{X_{(1)}}(x)=\frac{m !}{(i-1) !(m-i) !} f_{X_{(1)}}(x)\left[1-\frac{c(\theta G(x))}{c(\theta)}\right]^{i-1}\left[\frac{c(\theta G(x))}{c(\theta)}\right]^{m-i}, x>0 .
$$

This expression can be written as

$$
f^{X_{(1)}}(x)=\frac{m !}{(i-1) !(m-i) !} f_{X_{(1)}}(x) \sum_{j=0}^{i-1}(-1)^{j}\left(\begin{array}{c}
i-1 \\
j
\end{array}\right)\left[\frac{c(\theta G(x))}{c(\theta)}\right]^{m-j-1}, x>0 .
$$

\section{Estimation and inference}

Let $X_{1}, \ldots, X_{n}$ be a random sample, with the observed values $x_{1}, \ldots, x_{n}$ obtained from the GEWPS with parameters $\theta, \alpha, \delta$ and $\xi$. Let $\Theta=(\theta, \alpha, \delta, \xi)$ be the $p \times 1$ parameter vector. The log likelihood function is given by

$$
\begin{aligned}
\ln = & n[\log \theta+\log \alpha+\log \delta-\log (\mathrm{c}(\theta))]-\alpha \sum_{i=1}^{n} H\left(x_{i}\right)+(\delta-1) \sum_{i=1}^{n} \log \left(1-e^{-\alpha H\left(x_{i}\right)}\right) \\
& +\sum_{i=1}^{n} \log \left(\mathrm{c}^{\prime}\left(\theta\left(1-e^{-\alpha H\left(x_{i}\right)}\right)^{\delta}\right)\right)
\end{aligned}
$$

Consider $p_{i}=1-e^{-\alpha H\left(x_{i} ; \eta\right)}$. Then the score function is given by $U_{n}(\Theta)=(\partial \ln / \partial \theta, \partial \ln / \partial \alpha, \partial \ln / \partial \delta, \partial \ln / \partial \xi)^{T}$. 


$$
\begin{aligned}
& \frac{\partial \ln }{\partial \theta}=\frac{n}{\theta}+\sum_{i=1}^{n} \frac{p_{i}{ }^{\delta} c^{\prime \prime}\left(\theta p_{i}{ }^{\delta}\right)}{c^{\prime}\left(\theta p_{i}{ }^{\delta}\right)}-n \frac{c^{\prime}(\theta)}{c(\theta)} \\
& \frac{\partial \ln }{\partial \alpha}=\frac{n}{\alpha}-\sum_{i=1}^{n} H\left(x_{i}\right)+(\delta-1) \sum_{i=1}^{n} \alpha h\left(x_{i}\right) \frac{\left(1-p_{i}\right)}{p_{i}}+\sum_{i=1}^{n} \frac{\theta \delta H\left(x_{i}\right)\left(1-p_{i}\right) p_{i}{ }^{\delta-1} c^{\prime \prime}\left(\theta p_{i}{ }^{\delta}\right)}{c^{\prime}\left(\theta p_{i}{ }^{\delta}\right)} \\
& \frac{\partial \ln }{\partial \delta}=\frac{n}{\delta}+\sum_{i=1}^{n} \log \left(p_{i}\right)+\sum_{i=1}^{n} \frac{\theta p_{i}^{\delta} \log \left(p_{i}\right)\left(c^{\prime \prime}\left(\theta p_{i}{ }^{\delta}\right)\right.}{c^{\prime}\left(\theta p_{i}{ }^{\delta}\right)} \\
& \frac{\partial \ln }{\partial \xi_{k}}=\sum_{i=1}^{n} \frac{\partial \operatorname{logh}\left(x_{i}\right)}{\partial \xi_{k}}-\alpha \sum_{i=1}^{n} \frac{\partial H\left(x_{i}\right)}{\partial \xi_{k}}\left[1+(\delta-1) \frac{1-p_{i}}{p_{i}}+\theta \delta\left(1-p_{i}\right) p_{i}{ }^{\delta-1} \frac{c^{\prime \prime}\left(\theta p_{i}{ }^{\delta}\right)}{c^{\prime}\left(\theta p_{i}{ }^{\delta}\right)}\right]
\end{aligned}
$$

The maximum likelihood estimation (MLE) of $\Theta$ say $\Theta$ is obtained by solving the nonlinear system $U_{n}(\mathrm{x} ; \Theta)=0$. This nonlinear system of equations does not have a closed form. For the interval estimation and hypothesis tests on the model parameters, we require the following observed information matrix:

$$
I_{n}(\Theta)=-\left[\begin{array}{ccccc}
I_{\theta \theta} & I_{\theta \alpha} & I_{\theta \delta} & \mid & I_{\theta \xi}^{T} \\
I_{\alpha \theta} & I_{\alpha \alpha} & I_{\alpha \delta} & \mid & I_{\alpha \xi}^{T} \\
I_{\delta \theta} & I_{\delta \alpha} & I_{\delta \delta} & \mid & I_{\delta \xi}^{T} \\
- & - & - & - & - \\
I_{\theta \xi} & I_{\alpha \xi} & I_{\delta \xi} & \mid & I_{\xi \xi}
\end{array}\right],
$$

where the elements of $I_{n}(\Theta)$ are the second partial derivatives of $U_{n}(\Theta)$. Under the standard regular conditions for the large sample approximation (Cox and Hinkley, 1974) fulfilled for the proposed model, the distribution of $\Theta$ is approximately $N_{p}\left(\Theta, J_{n}(\Theta)^{-1}\right)$, where $J_{n}(\Theta)=\mathrm{E}\left[\mathrm{I}_{n}(\Theta)\right]$. Whenever the parameters are in the interior of the parameter space but not on the boundary, the asymptotic distribution of $\sqrt{n}(\Theta-\Theta)$ is $N_{p}\left(0, J(\Theta)^{-1}\right)$, where $J(\Theta)^{-1}=\lim _{n \rightarrow \infty} n^{-1} I_{n}(\Theta)$ is the unit information matrix and $p$ is the number of parameters of the distribution. The asymptotic multivariate normal $N_{p}\left(\Theta, I_{n}(\Theta)^{-1}\right)$ distribution of $\Theta$ can be used to approximate the confidence interval for the parameters, the hazard rate, and the survival functions. An $100(1-\gamma)$ asymptotic confidence interval for parameter $\Theta_{i}$ is given by

$$
\left(\Theta_{i}-Z_{\frac{\gamma}{2}} \sqrt{I^{i i}}, \Theta_{i}+Z_{\frac{\gamma}{2}} \sqrt{I^{i i}}\right),
$$

where $I^{i i}$ is the $(i, i)$ diagonal element of $I_{n}(\Theta)^{-1}$ for $i=1, \ldots, p$ and $Z_{\frac{\gamma}{2}}$ is the quantile $1-\gamma / 2$ of the standard normal distribution. 


\subsection{EM algorithm}

An insurance company promises to pay claims to the insureds if some defined events (injury, accident, death, etc.) occur. However in many cases, claims originating in a particular year are often settled with a time delay of years or perhaps decades. Therefore, a method to estimate the expected liability is needed so that the insurer can calculate the profit of written policies, and allocate reserved assets to ensure liquidity. Since loss reserves generally represent by far the largest liability, and the greatest source of financial uncertainty in an insurance company, an appropriate valuation of insurance liabilities including risk margin is one of the most important issues for a general insurer. Risk margin is the component of the value of claims liability that relates to the inherent uncertainty.

Based on the underline distribution, the MLE of the parameters can be found analytically using the EM algorithm. The Newton-Raphson algorithm is one of the standard methods to determine the MLEs of the parameters. To use the algorithm, the second derivatives of the loglikelihood are required for all iterations. The EM algorithm is a very powerful tool for handling the incomplete data problem (Dempster et al., 1977; McLachlan and Krishnan, 1983). It is an iterative method that repeatedly replaces missing data with estimated values and updates the parameter estimates. It is especially useful if the complete dataset is easy to analyze. As pointed out by Little and Rubin (1983), the EM algorithm converge reliably but rather slowly, compared with the Newton-Raphson method, when the amount of information in the missing data is relatively large. Recently, EM algorithm has been used by such researchers as Adamidis and Loukas (1998), Adamidis (1999), Ng et al. (2002), Karlis (2003), and Adamidis et al. (2005).

In estimating $\Theta$, the EM algorithm is a recurrent method in which each step consists of an estimate of the expected value of a hypothetical random variable and later maximizes the loglikelihood of the complete data. Let the complete data be $X_{1}, \ldots, X_{n}$, with the observed values $x_{1}, \ldots, x_{n}$ and the hypothetical random variable $N_{1}, \ldots, N_{n}$. The joint probability function is such that the marginal density of $X_{1}, \ldots, X_{n}$ is the likelihood of interest. Then, we define a hypothetical complete data distribution for each $\left(X_{i}, N_{i}\right)^{T}, i=1, \ldots, \mathrm{m}$, with a joint probability function in the form of

$$
\mathrm{f}_{\mathrm{X}, \mathrm{N}}(\mathrm{x}, \mathrm{z} ; \Theta)=\frac{a_{z} \theta^{z}}{c(\theta)} z \alpha \delta h(x) e^{-\alpha H(x)}\left(1-e^{-\alpha H(x)}\right)^{z \delta-1},
$$

where $x, \mathscr{\theta} \delta, \alpha \in \mathrm{R}^{+}$and $z \in \mathrm{N}$. Therefore, it is straightforward to verify that the E-step of an EM cycle requires the computation of the conditional expectation of $\left(\mathrm{Z} \mid \mathrm{X} ; \Theta^{(r)}\right)$, where $\Theta^{(r)}=\left(? \theta^{(r)}, \alpha^{(r)}, \delta^{(r)}, \xi^{(r)}\right.$ is the current estimate (in the rth iteration) of $\Theta$. Then, the EM cycle is completed with the M-step, which is complete data maximum likelihood over $(\Theta)$, with the missing Z's replaced by their conditional expectations $E(\mathrm{Z} \mid \mathrm{X} ; \Theta)$ (Adamidis and Loukas, 1998), where 


$$
p_{Z \mid X}(z)=\frac{\mathrm{f}_{\mathrm{X}, \mathrm{N}}(\mathrm{x}, \mathrm{z} ; \Theta)}{f_{X}(x)}=\frac{a_{z} \theta^{z-1} z\left(1-e^{-\alpha H(x)}\right)^{z \delta-\delta}}{c^{\prime}\left(\theta\left(1-e^{-\alpha H(x)}\right)^{\delta}\right)},
$$

and since $c^{\prime}(\theta)+c^{\prime \prime}(\theta)=\sum_{z=1}^{\infty} z^{2} a_{z} \theta^{z-1}$ its expected value is

$$
\begin{aligned}
E(Z \mid X) & =\sum_{z=1}^{\infty} \frac{a_{z} \theta^{z-1} z^{2}\left(1-e^{-\alpha H(x)}\right)^{z \delta-\delta}}{c^{\prime}\left(\theta\left(1-e^{-\alpha H(x)}\right)^{\delta}\right)}=\frac{1}{c^{\prime}\left(\theta\left(1-e^{-\alpha H(x)}\right)^{\delta}\right)} \sum_{z=1}^{\infty} z^{2} a_{z}\left[\theta\left(1-e^{-\alpha H(x)}\right)^{\delta}\right]^{z-1} \\
& =\frac{c^{\prime}\left(\theta\left(1-e^{-\alpha H(x)}\right)^{\delta}\right)+\theta\left(1-e^{-\alpha H(x)}\right)^{\delta} c^{\prime \prime}\left(\theta\left(1-e^{-\alpha H(x)}\right)^{\delta}\right)}{c^{\prime}\left(\theta\left(1-e^{-\alpha H(x)}\right)^{\delta}\right)} \\
& =1+\frac{\theta\left(1-e^{-\alpha H(x)}\right)^{\delta} c^{\prime \prime}\left(\theta\left(1-e^{-\alpha H(x)}\right)^{\delta}\right)}{c^{\prime}\left(\theta\left(1-e^{-\alpha H(x)}\right)^{\delta}\right)}
\end{aligned}
$$

\section{Special subclasses}

In this section, we present four special subclasses of the GEWPS family of distributions. We provide the forms of the cumulative, density survival, and hazard rate functions for $X_{(1)}$ and $X_{(\mathrm{n})}$.

\subsection{A compound class of the Poisson and lifetime distributions}

The compound class of the Poisson distribution (CP) (Alkarni and Oraby, 2012) is a subclass of the GEWPS family of distribution, with $a_{n}=\frac{1}{n !}, c(\theta)=e^{\theta}-1,(\theta>0)$. We assume that $X_{1}, \ldots, X_{N}$ are identically independent random variables with a distribution function as in (3) and with $N$ following a truncated Poisson distribution at zero. Table 2 shows the necessary functions for this class.

Table 2: Cdf, pdf, survival, and hazard rate functions for the CL class

\begin{tabular}{|l|l|}
\hline$X_{(1)}=\min \left(X_{1}, \ldots, X_{N}\right)$ & $X_{(\mathrm{n})}=\max \left(X_{1}, \ldots, X_{N}\right)$ \\
\hline$F(x)=\frac{1-e^{-\theta \mathrm{G}(\mathrm{x})}}{1-e^{-\theta}}$ & $F(x)=\frac{e^{-\theta(1-\mathrm{G}(\mathrm{x}))}-e^{-\theta}}{1-e^{-\theta}}$ \\
$f(x)=\frac{\theta g(x) e^{-\theta \mathrm{G}(\mathrm{x})}}{1-e^{-\theta}}$ & $f(x)=\frac{\theta g(x) e^{-\theta(1-\mathrm{G}(\mathrm{x}))}}{1-e^{-\theta}}$ \\
\hline
\end{tabular}




\begin{tabular}{|l|l|}
\hline$s(x)=\frac{e^{-\theta \mathrm{G}(\mathrm{x})}-e^{-\theta}}{1-e^{-\theta}}$ & $s(x)=\frac{1-e^{e^{-\theta(1-\mathrm{G}(\mathrm{x}))}}}{1-e^{-\theta}}$ \\
$\tau(x)=\frac{\theta g(x) e^{-\theta \mathrm{G}(\mathrm{x})}}{e^{-\theta \mathrm{G}(\mathrm{x})}-e^{-\theta}}$ & $\tau(x)=\frac{\theta g(x) e^{e^{-\theta(1-\mathrm{G}(\mathrm{x}))}}}{1-e^{e^{-\theta(1-\mathrm{G}(\mathrm{x}))}}}$ \\
\hline
\end{tabular}

\subsection{A compound class of logarithmic and lifetimes distributions}

The compound class of logarithmic distribution (CL) (Alkarni, 2012) is a subclass of the GEWPS family of distribution, with $a_{n}=1, c(\theta)=-\log (1-\theta), \theta \in(0,1)$. We assume that $X_{1}, \ldots, X_{N}$ are identically independent random variables with a distribution function as in (3) and with $N$ following a truncated logarithmic distribution at zero. Table 3 shows the necessary functions for this class.

Table 3: Cdf, pdf, survival, and hazard rate functions for the CL class

\begin{tabular}{|l|l|}
\hline$X_{(1)}=\min \left(X_{1}, \ldots, X_{N}\right)$ & $X_{(\mathrm{n})}=\max \left(X_{1}, \ldots, X_{N}\right)$ \\
\hline$F(x)=1-\frac{\log (1-\theta(1-G(x)))}{\log (1-\theta)}$ & $F(x)=\frac{\log (1-\theta G(x))}{\log (1-\theta)}$ \\
$f(x)=\frac{-\theta g(x)}{(1-\theta(1-\mathrm{G}(\mathrm{x}))) \log (1-\theta)}$ & $f(x)=\frac{-\theta g(x)}{(1-\theta \mathrm{G}(\mathrm{x})) \log (1-\theta)}$ \\
$s(x)=\frac{\log (1-\theta(1-G(x)))}{\log (1-\theta)}$ & $s(x)=1-\frac{\log (1-\theta G(x))}{\log (1-\theta)}$ \\
$\tau(x)=\frac{-\theta g(x)}{\log (1-\theta(1-\mathrm{G}(\mathrm{x})))[1-\theta(1-G(x))]}$ & $\tau(x)=\frac{\theta g(x)}{(1-\theta \mathrm{G}(\mathrm{x}))[\log (1-\theta \mathrm{G}(\mathrm{x}))-\log (1-\theta)]}$ \\
\hline
\end{tabular}

\subsection{A compound class of geometric and lifetime distributions}

The compound class of geometric distribution (CG) (Alkarni, 2013) is a subclass of the GEWPS family of distribution, with $a_{n}=1, c(\theta)=\theta(1-\theta)^{-1}, \theta \in(0,1)$. We assume that $X_{1}, \ldots, X_{N}$ are identically independent random variables with a distribution function as in (3) and with $N$ following a truncated geometric distribution at zero. Table 4 shows the necessary functions for this class

Table 4: Cdf, pdf, survival, and hazard rate functions for the CG class 


\begin{tabular}{|l|l|}
\hline$X_{(1)}=\min \left(X_{1}, \ldots, X_{N}\right)$ & $X_{(\mathrm{n})}=\max \left(X_{1}, \ldots, X_{N}\right)$ \\
\hline$F(x)=1-\frac{(1-\theta)(1-\mathrm{G}(\mathrm{x}))}{1-\theta(1-G(x))}$ & $F(x)=\frac{(1-\theta) \mathrm{G}(\mathrm{x})}{1-\theta G(x)}$ \\
$f(x)=\frac{(1-\theta) g(x)}{(1-\theta(1-G(x)))^{2}}$ & $f(x)=\frac{(1-\theta) g(x)}{(1-\theta G(x))^{2}}$ \\
$s(x)=\frac{(1-\theta)(1-\mathrm{G}(\mathrm{x}))}{1-\theta(1-G(x))}$ & $s(x)=1-\frac{(1-\theta) \mathrm{G}(\mathrm{x})}{1-\theta G(x)}$ \\
$\tau(x)=\frac{g(x)}{(1-\theta(1-G(x)))(1-\mathrm{G}(\mathrm{x}))}$ & $\tau(x)=\frac{(1-\theta) g(x)}{(1-\theta G(x))(1-\mathrm{G}(\mathrm{x}))}$ \\
\hline
\end{tabular}

\subsection{A compound class of binomial and lifetime distributions}

The compound class of binomial distribution (CB) (Alkarni, 2013) is a subclass of the GEWPS family of distributions with $c(\theta)=(\theta+1)^{m}-1$. We assume that $X_{1}, \ldots, X_{N}$ are identically independent random variables with a distribution function as in (3) and with $N$ following a truncated binomial distribution at zero. Table 5 shows the necessary functions for this class.

Table 5: Cdf, pdf, survival, and hazard rate functions for the CB class

\begin{tabular}{|l|l|}
\hline$X_{(1)}=\min \left(X_{1}, \ldots, X_{N}\right)$ & $X_{(\mathrm{n})}=\max \left(X_{1}, \ldots, X_{N}\right)$ \\
\hline$F(x)=1-\frac{(\theta(1-G(x))+1)^{m}-1}{(\theta+1)^{m}-1}$ & $F(x)=\frac{(\theta G(x)+1)^{m}-1}{(\theta+1)^{m}-1}$ \\
$f(x)=\frac{\mathrm{m} \theta \mathrm{g}(\mathrm{x})(\theta(1-G(x))+1)^{m-1}}{(\theta+1)^{m}-1}$ & $f(x)=\frac{\mathrm{m} \theta \mathrm{g}(\mathrm{x})(\theta G(x)+1)^{m-1}}{(\theta+1)^{m}-1}$ \\
$s(x)=\frac{(\theta(1-G(x))+1)^{m}-1}{(\theta+1)^{m}-1}$ & $s(x)=1-\frac{(\theta G(x)+1)^{m}-1}{(\theta+1)^{m}-1}$ \\
$\tau(x)=\frac{\mathrm{m} \theta \mathrm{g}(\mathrm{x})(\theta(1-G(x))+1)^{m-1}}{(\theta(1-G(x))+1)^{m}-1}$ & $\tau(x)=\frac{\mathrm{m} \theta \mathrm{g}(\mathrm{x})(\theta G(x)+1)^{m-1}}{(\theta+1)^{m}-(\theta G(x)+1)^{m}}$ \\
\end{tabular}

Table 6 illustrates examples of some existing distributions with $H(x)$ and $c(\theta)$. The other functions can be obtained directly from the previous tables. 
Table 6: Special distributions with cdf and the corresponding $H(x)$ and $c(\theta)$

\begin{tabular}{|c|c|c|c|c|c|c|}
\hline Distribution & $H(x)$ & $\delta$ & $a_{n}$ & $c(\theta)$ & $F_{X_{(n)}}(x)$ & Reference \\
\hline Generalized geometric exponential & $x$ & $\delta$ & 1 & $\frac{\theta}{1-\theta}$ & $\frac{(1-\theta)\left(1-e^{-\alpha x}\right)^{\delta}}{1-\theta\left(\left(1-e^{-\alpha x}\right)^{\delta}\right.}$ & Mahmoudi and Jafari (2012) \\
\hline Exponentiated Weibull-geometric & $(\beta x)^{\gamma}$ & $\delta$ & 1 & $\frac{\theta}{1-\theta}$ & $\frac{(1-\theta)\left(1-e^{-(\beta x)^{\gamma}}\right)^{\delta}}{1-\theta\left(\left(1-e^{-(\beta x)^{\gamma}}\right)^{\delta}\right.}$ & Mahmoudi and Shiran (2012) \\
\hline Generalized Poisson exponential & $x$ & $\delta$ & $\frac{1}{n !}$ & $e^{\theta}-1$ & $\frac{e^{\theta}\left(1-e^{-\alpha x}\right)^{\delta}-1}{e^{\theta}-1}$ & Mahmoudi and Jafari (2012) \\
\hline Exponentiated Weibull Poisson & $(\beta x)^{\gamma}$ & $\delta$ & $\frac{1}{n !}$ & $e^{\theta}-1$ & $\frac{e^{\theta}\left(1-e^{-(\beta x)^{\gamma}}\right)^{\delta}-1}{e^{\theta}-1}$ & Mahmoudi and Sepahdar (2013) \\
\hline Generalized binomial exponential & $x$ & $\delta$ & $\left(\begin{array}{l}\mathrm{m} \\
\mathrm{n}\end{array}\right)$ & $(\theta+1)^{m}-1$ & $\frac{\left(\theta\left(1-e^{-\alpha x}\right)^{\delta}+1\right)^{m}-1}{(\theta+1)^{m}-1}$ & Mahmoudi and Jafari (2012) \\
\hline Generalized logarithmic exponential & $x$ & $\delta$ & $\frac{1}{n}$ & $-\log (1-\theta)$ & $\frac{\log \left(1-\theta\left(1-e^{-\alpha x}\right)^{\delta}\right)}{\log (1-\theta)}$ & Mahmoudi and Jafari (2012) \\
\hline Exponentiated Weibull-logarithmic & $(\beta x)^{\gamma}$ & $\delta$ & $\frac{1}{n}$ & $-\log (1-\theta)$ & $\frac{\log \left(1-\theta\left(1-e^{-(\beta x)^{\gamma}}\right)^{\delta}\right)}{\log (1-\theta)}$ & Mahmoudi et al. (2014) \\
\hline Pareto Poisson & $\log (x / k)$ & 1 & 1 & $e^{\theta}-1$ & $1-\frac{e^{\theta(k / x)^{\alpha}}-1}{e^{\theta}-1} ; x \geq k$ & Silva et al. (2013) \\
\hline Poisson-Lomax & $(1+\beta x)^{-\alpha}$ & 1 & 1 & $e^{\theta}-1$ & $\frac{e^{\theta(1+\beta x)^{-\alpha}}-1}{e^{\theta}-1}$ & Al-Zahrani and Sagor (2014) \\
\hline
\end{tabular}




\section{Submodels and applications}

In this section, two models are discussed with real data as examples of the GEWPS family. Geometric exponential distribution (GE) and generalized exponential geometric (GEG) distribution are fitted for real data. By substituting directly in the forms found in Table 4 from Section 6 for $X_{(n)}=\max \left\{X_{i}\right\}_{i=1}^{N}$, we obtain the following pdfs and hazard functions:

$$
\begin{aligned}
& f_{G E}(x ; \theta, \beta)=\frac{(1-\theta) \beta e^{-\beta x}}{\left[1-\theta\left(1-e^{-\beta x}\right)\right]^{2}} ; \beta, x>0 ; \theta \in(0,1), \\
& \tau_{G E}(x ; \theta, \beta)=\frac{\beta(1-\theta)}{1-\theta\left(1-e^{-\beta x}\right)} ; \beta, x>0 ; \theta \in(0,1), \\
& f_{G E G}(x ; \theta, \beta, \delta)=\frac{(1-\theta) \beta \delta e^{-\beta x}\left(1-e^{-\beta x}\right)^{\delta-1}}{\left[1-\theta\left(1-e^{-\beta x}\right)^{\delta}\right]^{2}} ; \beta, \delta, x>0 ; \theta \in(0,1), \\
& \tau_{G E G}(x ; \theta, \beta, \delta)=\frac{(1-\theta) \beta \delta e^{-\beta x}\left(1-e^{-\beta x}\right)^{\delta-1}}{\left(1-\theta\left(1-e^{-\beta x}\right)^{\delta}\right)\left(1-\left(1-e^{-\beta x}\right)^{\delta}\right)} ; \beta, \delta, x>0 ; \theta \in(0,1) .
\end{aligned}
$$

Figs. 1 and 2 show the densities and hazard functions of the GE and GEG distributions for the selected parameter values. 

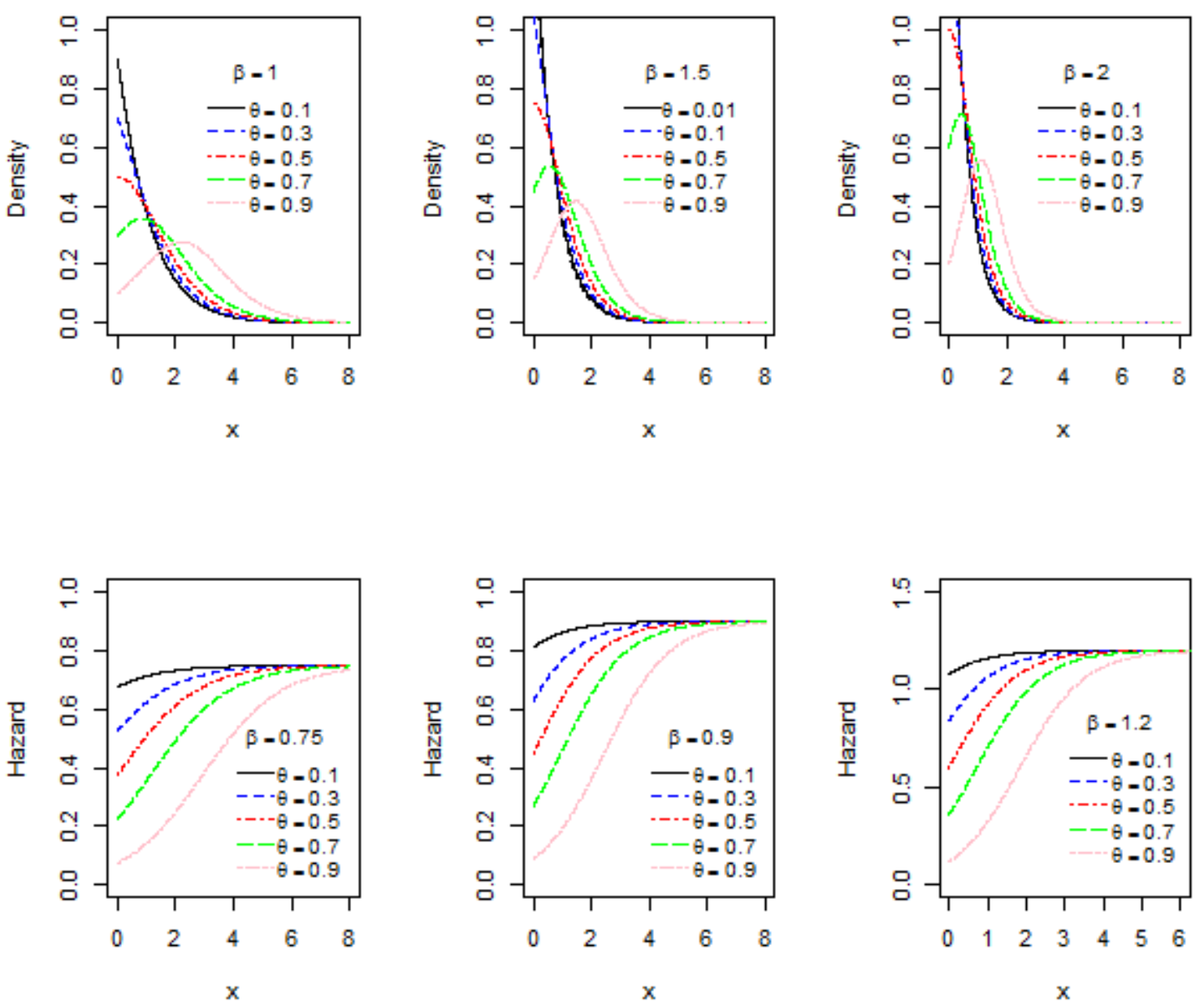

Fig. 1. : Plots of the density and hazard rate function of the GE for different values of $\beta$ and $\theta$. 

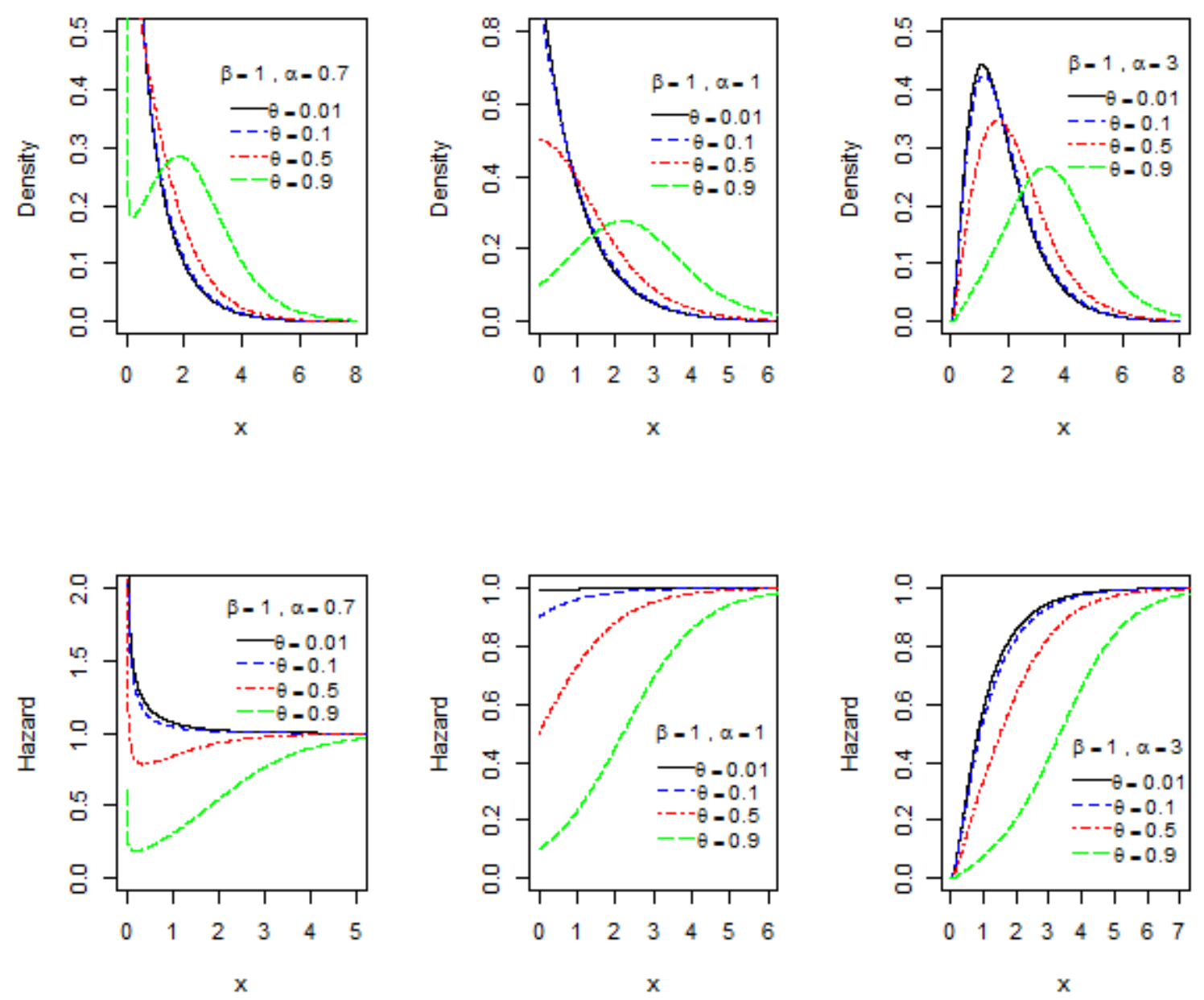

Fig. 2. : Plots of the density and hazard rate function of the GEG for $\beta=1$ and different values $\delta$ and $\boldsymbol{\theta}$.

Both models are fitted for two real data sets. The first set was introduced by Birnbaum and Saunders (1969) on the fatigue life of 6061-T6 aluminum coupons cut parallel with the direction of rolling and oscillated at 18 cycles per second. The data are listed in Table 7, which consists of 101 observations.

Table 7 Fatigue life of 6061-T6 aluminum coupons 


\begin{tabular}{ccccccccccccccc}
\hline 70 & 90 & 96 & 97 & 99 & 100 & 103 & 104 & 104 & 105 & 107 & 108 & 108 & 108 & 109 \\
109 & 112 & 112 & 113 & 114 & 114 & 114 & 116 & 119 & 120 & 120 & 120 & 121 & 121 & 123 \\
124 & 124 & 124 & 124 & 124 & 128 & 128 & 129 & 129 & 130 & 130 & 130 & 131 & 131 & 131 \\
131 & 131 & 132 & 132 & 132 & 133 & 134 & 134 & 134 & 134 & 134 & 136 & 136 & 137 & 138 \\
138 & 138 & 139 & 139 & 141 & 141 & 142 & 142 & 142 & 142 & 142 & 142 & 144 & 144 & 145 \\
146 & 148 & 148 & 149 & 151 & 151 & 152 & 155 & 156 & 157 & 157 & 157 & 157 & 158 & 159 \\
162 & 163 & 163 & 164 & 166 & 166 & 168 & 170 & 174 & 196 & 212 & & & &
\end{tabular}

The EM algorithm is used to estimate the model parameters. The MLEs of the parameters, the maximized log likelihood, the Kolmogorov-Smirnov statistics with its respective p-value and the Akaike Information Criterion (AIC) for the GE and GEG models are given in Table 8. The K-S test is valid to test the fit of underlying distribution to the failure data (Bagheri et al. 2016). The fitted densities and the empirical distribution versus the fitted cdfs of the GE and GEG models of this data are shown in Fig. 3. They indicate that the GEG distribution fits the data better than the GE distribution. The KS test statistic takes the smallest value with the largest value of its corresponding p-value for the GEG distribution. Moreover, this conclusion is confirmed from the log likelihood and the AIC for the fitted models given in Table 8. Their densities and cumulative distributions are plotted in Fig. 4.

Table 8: Parameter estimates, KS statistic, P-value, log likelihood and AIC of the Birnbaum and Saunders data.

\begin{tabular}{|c|c|c|c|c|c|}
\hline Dist. & MLE(std.) & $\mathrm{K}-\mathrm{S}$ & $\mathrm{p}$-value & $-\log (\mathrm{L})$ & AIC \\
\hline GE & $\begin{array}{l}\hat{\theta}=0.9999 \\
\hat{\beta}=0.0695\end{array}$ & 0.076 & 0.6041 & 457.1 & 918.3 \\
\hline GEG & $\begin{array}{l}\hat{\theta}=0.9884 \\
\hat{\beta}=0.0795 \\
\hat{\delta}=464.085\end{array}$ & 0.0492 & 0.9673 & 455.2 & 916.4 \\
\hline
\end{tabular}



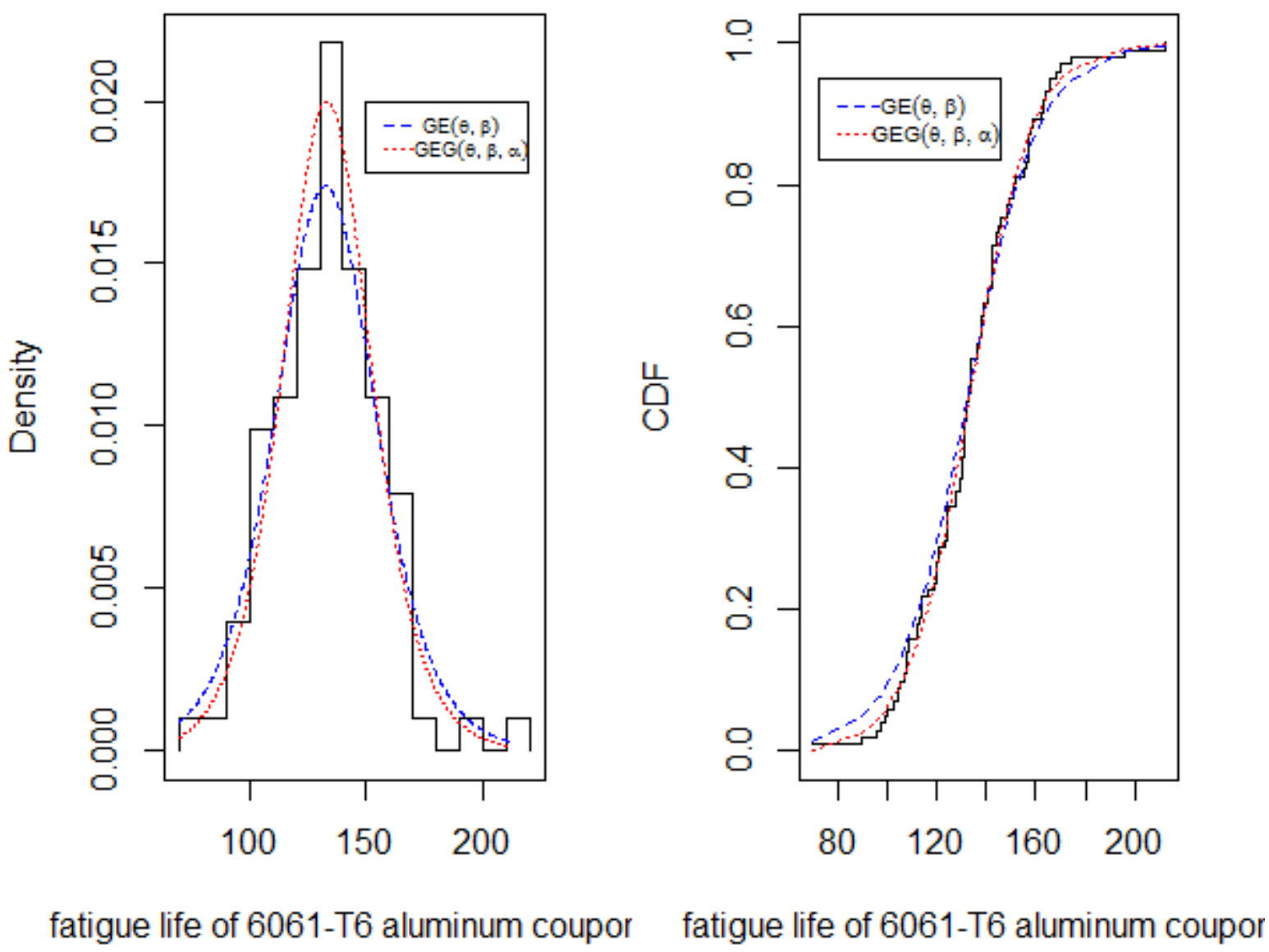

fatigue life of 6061-T6 aluminum coupor

fatigue life of 6061-T6 aluminum coupor

Fig. 3. : Plots of fitted GEG and GE of the Birnbaum and Saunders data.

The second data set represent the fatigue life for 67 specimens of Alloy T7987 that failed before having accumulated 300 thousand cycles of testing. This data was recently analyzed by Mahmoudi and Jafari (2012). The data are listed in Table 9.

Table 9: Fatigue life of 6061-T6 aluminum coupons 


\begin{tabular}{cllllllllllllll}
\hline 94 & 96 & 99 & 99 & 104 & 108 & 112 & 114 & 117 & 117 & 118 & 121 & 121 & 123 & 129 \\
131 & 133 & 135 & 136 & 139 & 139 & 140 & 141 & 141 & 143 & 144 & 149 & 149 & 152 & 153 \\
159 & 159 & 159 & 159 & 162 & 168 & 168 & 169 & 170 & 170 & 171 & 172 & 173 & 176 & 177 \\
180 & 180 & 184 & 187 & 188 & 189 & 190 & 196 & 197 & 203 & 205 & 211 & 213 & 224 & 226 \\
227 & 256 & 257 & 269 & 271 & 274 & 291 & & & & & & & &
\end{tabular}

The EM algorithm is used to estimate the model parameters. The MLEs of the parameters, the maximized log likelihood, the Kolmogorov-Smirnov statistics with its respective $p$-value and the Akaike Information Criterion (AIC) for the GE and GEG models are given in Table 10. The fitted densities and the empirical distribution versus the fitted cdfs of the GE and GEG models of this data are shown in Fig. 4. They indicate that the GEG distribution fits the data better than the GE distribution. The KS test statistic takes the smallest value with the largest value of its corresponding p-value for the GEG distribution. Moreover, this conclusion is confirmed from the $\log$ likelihood and the AIC for the fitted models given in Table 8. Their densities and cumulative distributions are plotted in Fig. 4.

Table 10: Parameter estimates, KS statistics, P-value, log likelihood and AIC for Fatigue Life Data for Alloy T7987.

\begin{tabular}{cccccc}
\hline Dist. & MLE(std.) & K-S & p-value & $-\log (\mathrm{L})$ & AIC \\
\hline \multirow{2}{*}{ GE } & $\hat{\theta}=0.9979$ & 0.0673 & 0.9222 & 351.9 & 707.4 \\
& $\hat{\beta}=0.0381$ & & & \\
& & & & \\
& $\hat{\theta}=0.2873$ & & & \\
GEG & $\hat{\beta}=0.0286$ & 0.0585 & 0.9757 & 347.8 & 701.6 \\
& $\hat{\delta}=50.4547$ & & &
\end{tabular}



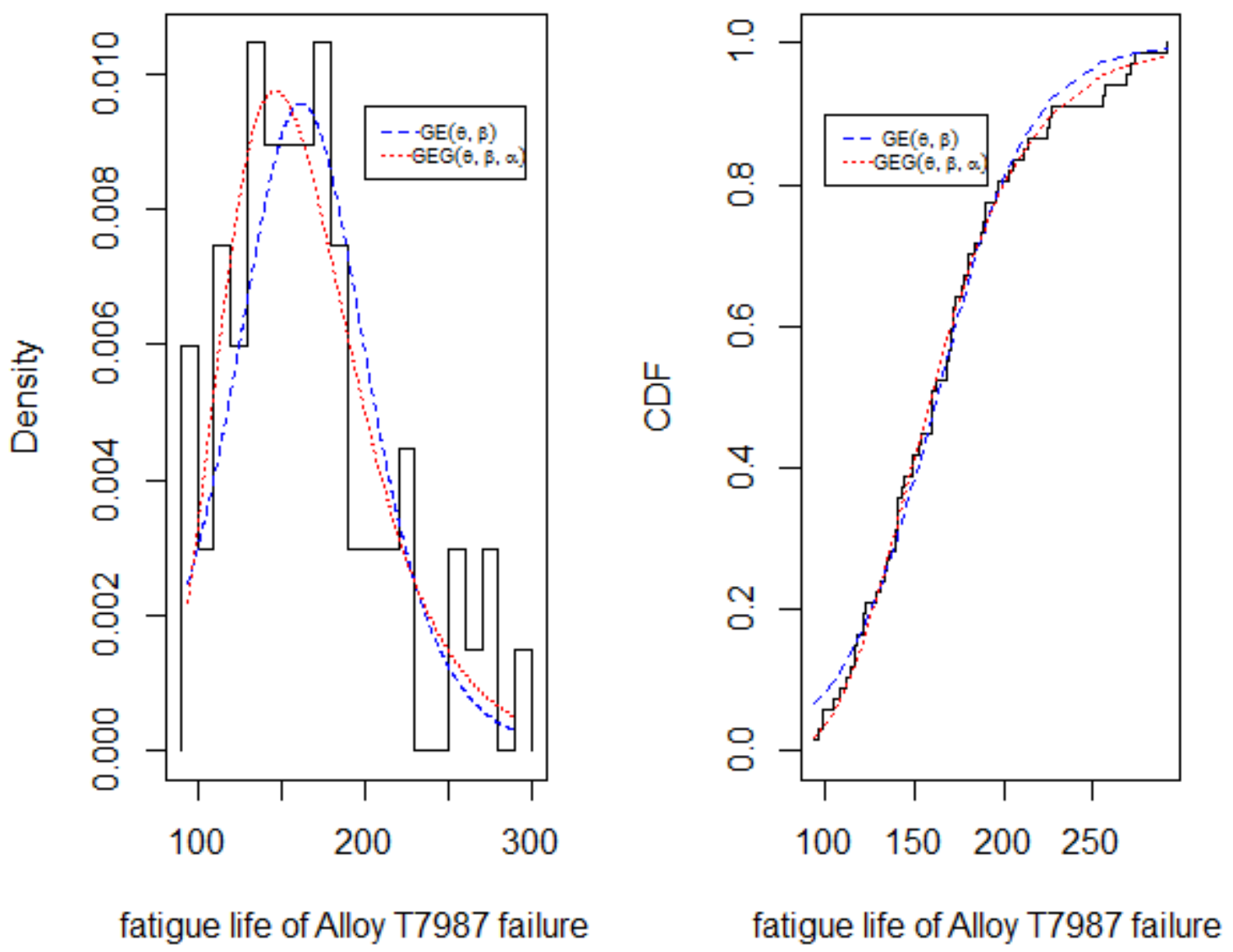

Fig. 4. : Plots of fitted GEG and GE of Fatigue Life Data for Alloy T7987.

\section{Concluding remarks}

We define a new family of lifetime distributions called the GEWPS family of distributions, which generalizes the extended Weibull power series class and the generalized power series exponential distributions introduced by Silva et al. (2013) and Mahmoudi and Jafari (2012), respectively. The GEWPS class contains many lifetime subclasses and distributions. Various standard mathematical properties were derived, such as density and survival, and hazard functions were introduced in flexible and useful forms. Parameter estimation using the EM 
algorithm was conducted using the maximum likelihood method. Finally, we fitted some of the GEWPS models to real data sets to show the flexibility and the benefits of the proposed class.

\section{Acknowledgments}

The author is grateful to the Deanship of Scientific Research at King Saud University, as represented by the Research Center of the College of Business Administration, for financially supporting this study.

\section{References}

[1] Alkarni, S. and Oraby, A. "A compound class of Poisson and lifetime distributions", J. Stat. Appl. Pro. 1, 45-51, 2012.

[2] Alkarni, S. "New family of logarithmic lifetime distributions", J. mathematics and statistics, 8(4), 435-440, 2012.

[3] Alkarni, S. "A compound class of geometric and lifetimes distributions", The open stat. and prob. journal 5, 1-5, 2013.

[4] Alkarni, S. "A class of truncated binomial lifetime distributions", Open journal of stat., 3, 305-311, 2013.

[5] Adamidis, K. "An EM algorithm for estimating negative binomial parameters", Austral, New Zealand

Statist., 41 (2), 213- 221, 1999.

[6] Adamidis, K., Dimitrakopoulou, T. and Loukas, S., "On an extension of the exponentialgeometric

Distribution", Statist. Probab. Lett., 73, 259-269, 2005.

[7] Adamidis, K. and Loukas, S., "A lifetime distribution with decreasing failure rate", Statistics and Probability Letters, 39, 35-42, 1998.

[8] Al-Zahrani, B. and Sagor, H., "The Poisson-lomax distribution", Revista Colombiana de Estadistica, 37(1), 225-245, 2014.

[9] Bagheri, S., Bahrani E. and Ganjali, M., The generalized modified Weibull power series distribution: Theory and applications", Computational statistics and data analysis, 94, 136$160,2016$.

[10]Birnbaum, Z. and Saunders, S., " Estimation for a family of life distributions with applications to fatigue", j. of applied prob., 6, 328-347, 1969.

[11]Bourguignon, M., Silva, R. and Cordeiro, G. "A new class of fatigue life distributions", Journal of statistical computation \&simulation, 84, 2619-2635, 2014.

[12]Chahkandi, M., Ganjali, M., 2009. On some lifetime distributions with decreasing failure rate. Computational

Statistics and Data Analysis 53, 4433-4440

[13]Cox, D. and Hinkley, D., "Theoretical Statistics ", Chapman and Hall, London, 1974. 
[14]Dempster, A., Laird, N. and Rubin, D., "Maximum likelihood from incomplete data via the EM

Algorithm ", J. Roy. Statist. Soc. Ser. B, 39, 1-38, 1977.

[15]Flores, Borges, J., Cancho, P. and Louzada, G., "The complementary exponential power series distribution", Brazilian Journal of Probability and Statistics, 27(4), 565-584, 2013.

[16]Johnson, N., Kotz, S. and Balakrishnan N., Continuous Univariate Distributions 2nd. ed., 1, Wiley,

New York, 1994.

[17]Gupta, R. and Kundu, D., " Generalized exponential distribution", Australian and New Zealand Journal of Statistics, 41, 173-188, 1999.

[18]Gupta, R. and Kundu, D. , " Generalized exponential distributions; Different Method of Estimations", $J$. Statist. Comput. and Simula., 69, 315-338, 2000.

[19]Gurvich, M., Dibenedetto, A. Ranade, S., " A new statistical distribution for characterizing the random strength of brittle materials, J. Mater. Sci., 32, 2559-2564, 1997.

[20]Karlis, D. "An EM algorithm for multivariate Poisson distribution and related models", J. Appl. Statist., 301, 63-77, 2003.

[21]Kus, C., 2007. A new lifetime distribution. Computational Statistics and Data Analysis 51, 4497-4509, 2007.

[22]Lai, C., Xie, M., and Murthy, D., "Modified Weibull model", IEEE Transactions on Reliability, 52, 33-37, 2003.

[23]Leahu, A., Munteanu B., and Cataranciuc, S. "On the lifetime as the maximum or minimum of the sample with power series distributed size", ROMAI jornal, 9, 119-128, 2013.

[24]Little, R. and Rubin, D., "Incomplete data. In: Kotz, S., Johnson, N.L. (Eds.), Encyclopedia of Statistical Sciences", 4, Wiley, NewYork,1983.

[25]Mahmoudi, E. and Jafari, A., "Generalized exponential- power series distributions", Computational Statistics and Data Analysis, 56, 4047-4066, 2012.

[26]Mahmoudi, E. and Sepahdar, A., " Exponentiated Weibull-Poisson distribution: model, properties and applications", Mathematics and computers in simulation, 92, 76-97, 2013.

[27]Mahmoudi, E., Sepahdar A. and Lemonte, A., "Exponentiated Weibull-logarithmic distribution: model, properties and applications", eprint arXiv:1402.5264, 02/2014.

[28]Mahmoudi, E. and Shiran, M., "Exponentiated Weibull power series distributions and its applications", eprint arXiv: $1212.5613,12 / 2012$. 
[29]Mahmoudi, E. and Shiran, M., "Exponentiated Weibull-geometric distribution and its applications",eprint arXiv: $1206.4008,06 / 2012$.

[30]McLachlan, G. and Krishnan, T., "The EM Algorithm and Extension", Wiley, New York, 1997.

[31]Morais, A. and Barreto-Souza, W., "A compound class of Weibull and power series distributions",

Computational Statistics and Data Analysis, 55, 1410-1425, 2011.

[32]Ng, M. Chan, P. and Balakrishnan, N., "Estimation of parameters from progressively censored data using EM algorithm", Comput. Statist. Data Anal., 39, 371-386, 2002.

[33]Peng, X. and Yan, Z., "Estimation and application for a new extended Weibull distribution", Reliability engineering and system safety, 121, 34-42, 2014.

[34]Rodriguez, R. , "A guide to the Burr Type XII distributions" ,Biometrika, 64, 129-134, 1977.

[35]Sarhan, A. and Apaloo, J., "Exponentiated modified Weibull extension distribution", Reliability Engineering and System Safety, 112, 137-144, 2013.

[36] Silva, B., Bourguignon, M., Dias, C. and Cordeiro, G., "The compound class of extended Weibull power series distributions", Computational Statistics and Data Analysis, 58, 352-367, 2013.

[37]Smith, R. and Bain, L., "An exponential power life-testing distribution. Communications in Statistics -

Theory and Methods", 4, 469-481, 1975.

[38]Tahmasbi, R., Rezaei, S.," A two-parameter lifetime distribution with decreasing failure rate", Computational Statistics and Data Analysis 52, 3889-3901, 2008.

[39]Xie, M., Tang, Y. and Goh, T., " A modified Weibull extension with bathtub-shaped failure rate function", Reliability Engineering and System Safety, 76, 279-285, 2002.

Received May 15, 2015; accepted November 02, 2015.

Said H. Alkarni

Department of Quantitative Analysis

King Saud University

King Saud University, Riyadh, Saudi Arabia

$\underline{\text { salkarni@ksu.edu.sa }}$ 
